\title{
The comprehensive analysis of DEG/ENaC subunits in Hydra reveals a large variety of peptide-gated channels, potentially involved in neuromuscular transmission
}

\author{
Marc Assmann ${ }^{1}$, Anne Kuhn², Stefan Dürrnagel1', Thomas W Holstein ${ }^{2}$ and Stefan Gründer ${ }^{* *}$
}

\begin{abstract}
Background: It is generally the case that fast transmission at neural synapses is mediated by small molecule neurotransmitters. The simple nervous system of the cnidarian Hydra, however, contains a large repertoire of neuropeptides and it has been suggested that neuropeptides are the principal transmitters of Hydra. An ion channel directly gated by Hydra-RFamide neuropeptides has indeed been identified in Hydra - the Hydra $\mathrm{Na}^{+}$ channel $(\mathrm{HyNaC}) 2 / 3 / 5$, which is expressed at the oral side of the tentacle base. Hydra-RFamides are more widely expressed, however, being found in neurons of the head and peduncle region. Here, we explore whether further peptide-gated HyNaCs exist, where in the animal they are expressed, and whether they are all gated by Hydra-RFamides.

Results: We report molecular cloning of seven new $\mathrm{HyNaC}$ subunits - HyNaC6 to HyNaC12, all of which are members of the DEG/ENaC gene family. In Xenopus oocytes, these subunits assemble together with the four already known subunits into thirteen different ion channels that are directly gated by Hydra-RFamide neuropeptides with high affinity (up to $40 \mathrm{nM}$ ). In situ hybridization suggests that HyNaCs are expressed in epitheliomuscular cells at the oral and the aboral side of the tentacle base and at the peduncle. Moreover, diminazene, an inhibitor of $\mathrm{HyNaCs}$, delayed tentacle movement in live Hydra.
\end{abstract}

Conclusions: Our results show that Hydra has a large variety of peptide-gated ion channels that are activated by a restricted number of related neuropeptides. The existence and expression pattern of these channels, and behavioral effects induced by channel blockers, suggests that Hydra co-opted neuropeptides for fast neuromuscular transmission.

Keywords: ASIC, DEG/ENaC, Evolution, Ligand-gated ion channel, Nervous system, Neuropeptide

\section{Background}

In general, fast neurotransmission is mediated by small molecule neurotransmitters acting on ionotropic receptors at the post-synaptic membrane, while neuropeptides bind G-protein-coupled receptors (GPCRs) and mediate slow neuromodulatory transmission. So far, there are only two exceptions to this general pattern: the Phe-Met-Arg-Pheamide (FMRFamide) activated $\mathrm{Na}^{+}$channel $(\mathrm{FaNaC})$ in

\footnotetext{
* Correspondence: sgruender@ukaachen.de

${ }^{1}$ Department of Physiology, RWTH Aachen University, D-52074 Aachen, Germany

Full list of author information is available at the end of the article
}

snails $[1,2]$ and the RF-amide activated $\mathrm{Hydra}^{+}{ }^{+}$channel $(\mathrm{HyNaC})[3,4]$. Both are ionotropic receptors, but their ligands are neuropeptides belonging to a family of FMRFamide-like peptides (FLPs) conserved from invertebrates to vertebrates [5]. Furthermore, $\mathrm{FaNaC}$ mediates fast responses of snail neurons to Phe-Met-Arg-Phe-amide (FMRFamide) [1,6-8], a common neuropeptide in many nervous systems [9].

$\mathrm{FaNaC}$ and $\mathrm{HyNaC}$ both belong to the degenerin/epithelial $\mathrm{Na}^{+}$channel (DEG/ENaC) gene family [10] but are not species orthologs. They rather belong to different branches of the DEG/ENaC family [3,4]. DEG/ENaCs 
are cation channels that are blocked by the diuretic amiloride and share sequence homology and a secondary structure characterized by two transmembrane spanning domains (TMDs) and a large extracellular domain (ECD). They have various functions. Degenerins from Caenorhabditis elegans are mechanosensitive channels that mediate touch transduction [11]. In contrast, $\mathrm{ENaC}$ is a constitutively open channel that mediates $\mathrm{Na}^{+}$reabsorption in the mammalian kidney [12]. Other family members from mammals are the acid-sensing ion channels (ASICs), ionotropic receptors for protons [13], and the bile-acid sensitive ion channel BASIC [14]. ASICs and BASIC are closely related to $\mathrm{HyNaCs}$ [3] and, interestingly, ASICs are modulated by direct binding of FLPs [15]. Pickpocket genes (PPKs) encode DEG/ENaCs in Drosophila and mediate diverse functions such as salt and water taste [16-18], mechanical nociception [19] and pheromone-sensing [20-22].

So far, four $\mathrm{HyNaC}$ subunits have been characterized: $\mathrm{HyNaC2}$ to $\mathrm{HyNaC5}$ [3,4]; hynac1 probably is a pseudogene because it lacks an initiator methionine [3]. It is now accepted that DEG/ENaCs are trimers [23-25]; while $\mathrm{FaNaC}$ is a homotrimer [2], $\mathrm{HyNaC}$ is a heterotrimer consisting of three different subunits, $\mathrm{HyNaC2}$, HyNaC3 and $\mathrm{HyNaC5}[3,4]$. hynac2, hynac3 and hynac5 are coexpressed at the base of the tentacles, most likely in epitheliomuscular cells. Hydra-RFamides are expressed in neurons from the hypostomal and the peduncle region of Hydra [26,27], where they are contained within large dense core vesicles in axon terminals of neurons contacting epitheliomuscular cells, suggesting that they contribute to neuromuscular transmission in Hydra [28,29]. Since tentacle curling is delayed by amiloride [4], it has been proposed that $\mathrm{HyNaC}$ is involved in movement of the tentacles [4]. While hynac2 and hynac3 are uniformly expressed at the tentacle base [3,4], hynac5 is restricted to the oral side [4], suggesting that $\mathrm{HyNaC2} / 3 / 5$ is active only in epitheliomuscular cells at the oral side of the tentacle base. In contrast, hynac4 localizes to the aboral side of the tentacles [3,4]. HyNaC4 does not assemble with $\mathrm{HyNaC2}$ and $\mathrm{HyNaC} 3$ in a functional channel [3], suggesting the existence of further $\mathrm{HyNaC}$ subunits at the aboral side of the tentacles.

Since Hydra belongs to the phylum Cnidaria, which is a sister phylum to all bilaterians, the distant relation of $\mathrm{FaNaC}$ and $\mathrm{HyNaC}$ suggests that the common ancestor to all DEG/ENaCs might have been a peptide-gated ion channel. Alternatively, peptide-gating might have evolved twice independently in the DEG/ENaC gene family. A more definite answer to this question needs a survey of the full repertoire of DEG/ENaCs in Hydra to establish whether all DEG/ENaCs in Hydra are peptide-gated channels. Moreover, the presence of many more neuropeptides in the Hydra nervous system [30] and the presence of the precursors that encode Hydra-RFamides I and II [27] at the peduncle region [31] raises the questions of the existence of $\mathrm{HyNaCs}$ that are gated by peptides other than Hydra-RFamides I and II or that are expressed at the peduncle and activated by Hydra-RFamides I and II.

Here, we report a comprehensive analysis of the DEG/ $\mathrm{ENaC}$ gene family in Hydra. We show that there is a surprising variety of $\mathrm{HyNaCs}$ and that, with one possible exception, $\mathrm{HyNaCs}$ are heterotrimers and are activated by Hydra-RFamides I and II. Some HyNaCs are expressed at the peduncle where they could mediate fast responses to RFamides I and II. Moreover, we establish the rules by which the DEG/ENaC subunits in Hydra assemble into functional peptide-gated channels.

\section{Results}

\section{The comprehensive analysis of DEG/ENaCs in Hydra}

We cloned seven new cDNAs coding for proteins with high homology to the known HyNaCs (see Methods). We named these proteins $\mathrm{HyNaC6}$ to $\mathrm{HyNaC12}$. They have a size of 454 to 507 amino acids and a predicted molecular mass of 52.9 to $58.4 \mathrm{kDa}$. In general, the amino acid sequence identity of all $\mathrm{HyNaCs}$ varies between $29 \%$ and $65 \%$. The exception is $\mathrm{HyNaC12}$, which is only $14 \%$ to $17 \%$ identical to other HyNaCs. In addition, $\mathrm{HyNaC} 3$ and $\mathrm{HyNaC} 11$ are $85 \%$ identical to each other. Phylogenetic analyses revealed that $\mathrm{HyNaC} 2$ to $\mathrm{HyNaC11}$ form a group of closely related channels within the DEG/ENaC gene family, suggesting that they derived from a single ancestor. $\mathrm{HyNaC6}$ and $\mathrm{HyNaC7}$ are more closely related to $\mathrm{HyNaC} 2$ and $\mathrm{HyNaC}$, whereas $\mathrm{HyNaC} 8$ to $\mathrm{HyNaC} 11$ are more closely related to $\mathrm{HyNaC3}$ and $\mathrm{HyNaC4}$, respectively, defining two subgroups within the $\mathrm{HyNaC}$ branch (highlighted by light and dark blue in Figure 1 and Additional file 1: Figure S1). Supporting previous results [3], $\mathrm{HyNaC2}$ to $\mathrm{HyNaC11}$ form a monophyletic group with ASIC and BASIC (see Figure 1 and Additional file 1: Figure S1). The grouping of $\mathrm{HyNaCs}$ and their relationship to ASICs and BASIC is apparent in the maximum likelihood analysis [see Additional file 1: Figure S1] and confirmed at a higher level of support by Bayesian analysis (Figure 1). The striking exception is $\mathrm{HyNaC12}$, which is isolated from other $\mathrm{HyNaCs}$ or ASICs and BASIC (Figure 1). While the genes coding for $\mathrm{HyNaC3}$ to $\mathrm{HyNaC11}$ have introns, the sequence coding for $\mathrm{HyNaC} 12$ is present as a single uninterrupted open reading frame in the Hydra magnipapillata genome, showing that hynac12 is intronless, and suggesting that it derived by retrotransposition early in cnidarian evolution. Retrotransposition is relatively common in Hydra [32].

The database contains the first 150 amino acids of another predicted protein that shows high homology to $\mathrm{HyNaCs}$ but like $\mathrm{HyNaC1}$ [3], $\mathrm{HyNaC13}$ lacked an initiator methionine and we were not able to clone it from Hydra cDNA. Therefore, we conclude that hynac13 is an inactive 


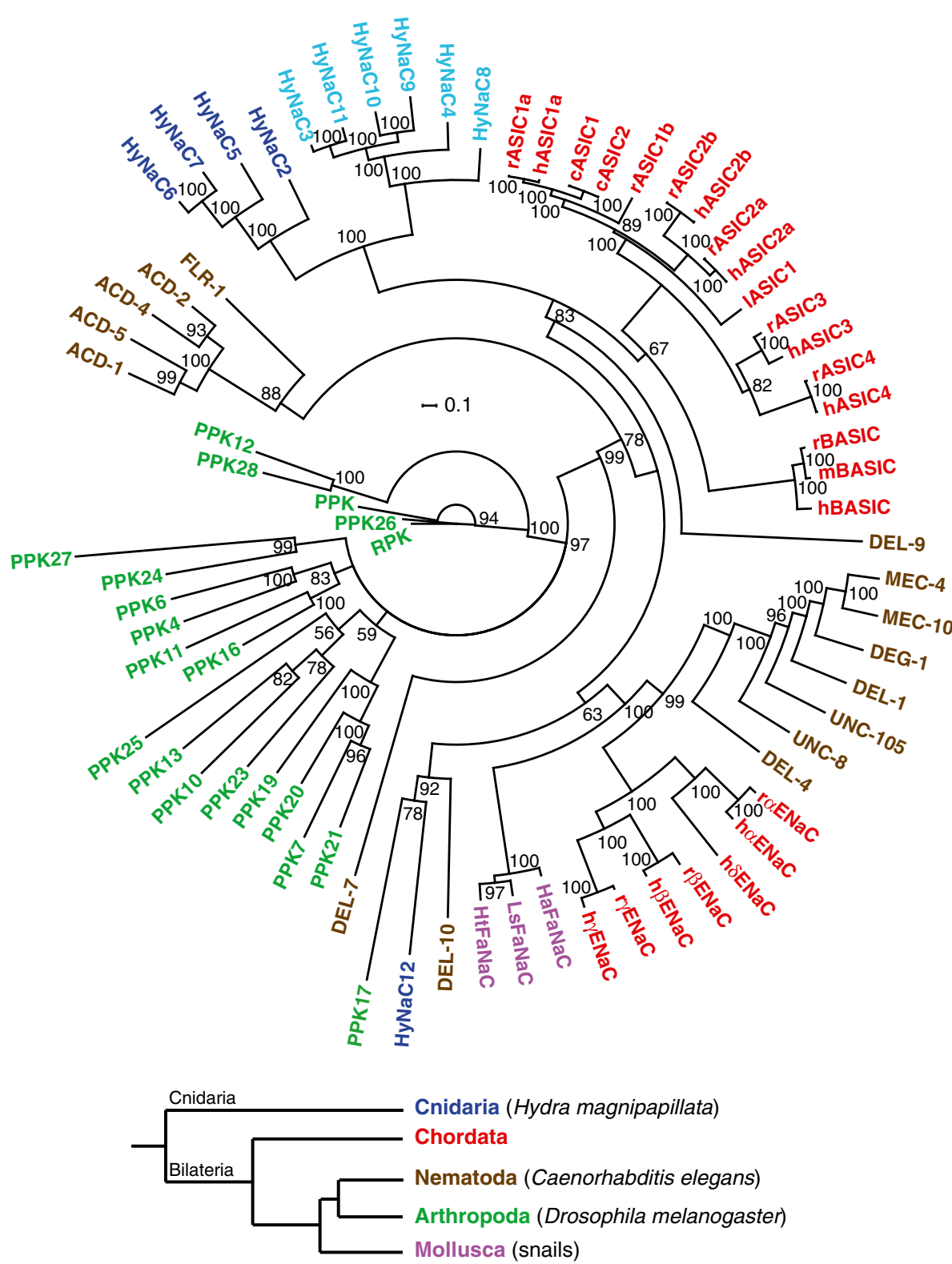

Figure 1 Consensus tree for the DEG/ENaC family. The phylogenetic tree was generated by Bayesian analysis with the program MrBayes 3.2 (see Methods). The numbers at nodes indicate the posterior probabilities computed by MrBayes for the respective node. Scale bar indicates amino acid exchanges per site. Colors indicate the following phyla: blue, Cnidaria; red, Chordata; green, Arthropoda; brown, Nematoda; pink, Mollusca. Abbrevations of species names are as follows: c, chicken (Gallus gallus); h, human (Homo sapiens); Ha, Helix aspersa; Ht, Helisoma trivolvis; I, lamprey (Lampetra fluviatilis), Ls, Lymnaea stagnalis; m, mouse (Mus musculus); $r$, rat (Rattus norvegicus). Other proteins are from C. elegans, Drosophila melanogaster or Hydra magnipapillata. Accession numbers are as follows: acid-sensitive degenerin (ACD)-1 [GenBank:NM_058894], ACD-2 [GenBank:NM_058892], ACD-4 [GenBank:NM_072829], ACD-5 [GenBank:NM_058795], CASIC1 [EMBL:AY956393], cASIC2 [GenBank:NM_001040467], hASIC1a [EMBL:U78180], hASIC2a [EMBL:U50352], hASIC2b [GenBank:NM_18337], hASIC3 [EMBL: AF095897], hASIC4 [EMBL:AJ271643], IASIC1 [EMBL:AAY28983], rASIC1a [EMBL:U94403], rASIC1b [EMBL:AJ309926], rASIC2a [EMBL:U5321 1], rASIC2b [EMBL: AB049451], rASIC3 [EMBL:AF013598], rASIC4 [EMBL:AJ271642], hBASIC [EMBL:AJ252011], mBASIC [EMBL:Y19035], rBASIC [EMBL:Y19034], DEG-1 NM_076910; degenerin-like (DEL)-1 [EMBL:U76403], DEL-4 [GenBank:NM_059829], DEL-7 [GenBank:NM_068875], DEL-9 [GenBank:NM_076221], DEL-10 [GenBank:NM-062901], ahENaC [EMBL:X76180], BhENaC [EMBL:X87159], YhENaC [EMBL:X87160], ShENaC [EMBL:U38254], arENaC [EMBL:X70521], BrENaC [EMBL:X77932], YrENaC [EMBL: X77933], HtFaNaC [EMBL:AF254118], LsFaNaC [EMBL:AF335548], HaFaNaC [EMBL:X92113], fluoride-resistant (FLR)-1 [EMBL:AB012617], HyNaC2 [EMBL:AM393878], HyNaC3 [EMBL:AM393880], HyNaC4 [EMBL:AM393881], HyNaC5 [EMBL:FN257513], HyNaC6 [EMBL:HG422725], HyNaC7 [EMBL:HG422726], HyNaC8 [EMBL: HG422727], HyNaC9 [EMBL:HG422728], HyNaC10 [EMBL:HG422729], HyNaC11 [EMBL:HG422730], HyNaC12 [EMBL:HG422731], mechanosensitive (MEC)-4 [EMBL: X58982], MEC-10 [EMBL:L25312], pickpocket (PPK) [EMBL:Y16225], PPK4 [GenBank:NM_206137], PPK6 [GenBank:NM_137617], PPK7 [GenBank:NM_135172], PPK10 [GenBank:NM_001038805], PPK11 [GenBank:NM_001038798], PPK12 [GenBank:NM_137828], PPK13 [GenBank:NM_001014495], PPK16 [GenBank:NM_001038797], PPK17 [GenBank:NM_135965], PPK19 [EMBL:AY226547], PPK20 [GenBank:NM_143448], PPK21 [GenBank:NM_143447], PPK23 [GenBank:NM_001014749], PPK24 [GenBank:NM_143603], PPK25 [GenBank:NM_206044], PPK26 [GenBank:NM_139868], PPK27 [GenBank:NM_139569], PPK28 [GenBank:NM_001014748], ripped pocket (RPK) [EMBL:Y12640], uncoordinated (UNC)-8 [EMBL:U76402], UNC-105 [GenBank:NM_063301]. 
pseudogene. Since extensive screening of the genomic database revealed no further $\mathrm{DEG} / \mathrm{ENaC}$ homologs, we conclude that $\mathrm{HyNaC} 2$ to $\mathrm{HyNaC12}$ constitute the whole DEG/ENaC gene family in Hydra magnipapillata.

All $\mathrm{HyNaCs}$ share conserved structures that are typical for DEG/ENaCs: they have short N- and C-termini, two TMDs and a large ECD between the TMDs [10]. Sequence motifs that are highly conserved in DEG/ENaCs are also at least partially present in $\mathrm{HyNaCs}$; examples are the N-terminal HG motif [33], a di-arginine motif right before TMD1, a highly conserved tryptophane in TMD1 and the selectivity filter motif 'GAS' in TMD2 [34,35]. Conserved cysteine residues in the ECD [36] indicate a conserved tertiary structure (Figure 2).

\section{New HyNaCs contribute to a variety of functional ion channels}

We tested the function of the new HyNaCs by coexpression of different $\mathrm{HyNaC}$ subunit combinations in Xenopus oocytes. We screened for functional subunit combinations by application of $5 \mu \mathrm{M}$ Hydra-RFamide I, a potent agonist of $\mathrm{HyNaC2} / 3 / 5$ [4]. When we expressed an individual $\mathrm{HyNaC}$ subunit we could never elicit currents. Similarly, the simultaneous expression of several cRNA never resulted in functional ion channels when $\mathrm{HyNaC} 2$ was absent. When $\mathrm{HyNaC} 2$ was part of the injected cRNA pool, however, Hydra-RFamide I elicited currents. Simultaneous expression of $\mathrm{HyNaC2}$ with a single other $\mathrm{HyNaC}$ resulted only in a functional channel when we co-expressed $\mathrm{HyNaC2}$ and $\mathrm{HyNaC} 3$; it was already known that $\mathrm{HyNaC2}$ and $\mathrm{HyNaC} 3$ form a lowaffinity peptide-gated channel [3]. Expression of $\mathrm{HyNaC2}$ with two other $\mathrm{HyNaCs}$, however, resulted in several functional ion channels (Figure 3).

Assembly of functional channels followed two simple rules. First, $\mathrm{HyNaC2}$ had to be present. Second, one of the other two subunits had to be from subgroup 1 (dark blue in Figure 1) containing $\mathrm{HyNaC5}$ to $\mathrm{HyNaC} 7$ and the other one from subgroup 2 (light blue in Figure 1) containing $\mathrm{HyNaC} 3, \mathrm{HyNaC} 4$ and $\mathrm{HyNaC} 8$ to $\mathrm{HyNaC11}$ as defined by phylogenetic relationship (Figure 1). Specifically, $\mathrm{HyNaC2}$ and $\mathrm{HyNaC5}$ formed functional heteromers with $\mathrm{HyNaC} 3$ or $\mathrm{HyNaC} 11$, while $\mathrm{HyNaC} 2$ and $\mathrm{HyNaC6}$ formed functional heteromers with all $\mathrm{HyNaCs}$ of the second subgroup. $\mathrm{HyNaC} 2$ and $\mathrm{HyNaC} 7$ formed functional heteromers with all members of the second subgroup except HyNaC8 (Figure 3).

The contribution of the distantly related $\mathrm{HyNaC} 12$ to peptide-gated channels was assessed by co-expression with $\mathrm{HyNaC} 2$ and one or several other $\mathrm{HyNaCs}(\mathrm{HyNaC} 3$ to $\mathrm{HyNaC11).} \mathrm{In} \mathrm{addition,} \mathrm{HyNaC12}$ was co-expressed with pools of different $\mathrm{HyNaCs}$ without $\mathrm{HyNaC2}$. In no case did this result in channels that could be activated by Hydra-RFamide I; in the case of $\mathrm{HyNaC} 2 / 3$, current amplitude and apparent peptide affinity were not increased by co-expression of $\mathrm{HyNaC12}$. We conclude that $\mathrm{HyNaC12}$ does not co-assemble with other $\mathrm{HyNaCs}$ into channels activated by Hydra-RFamide I. Since the closely related BASIC can efficiently be opened by lowering the concentration of extracellular divalent cations [37], reduction of extracellular divalent cation concentrations provides a potential means to open $\mathrm{HyNaCs}$ independently of specific agonists. In fact, all $\mathrm{HyNaCs}$ that were activated by Hydra-RFamide I were opened by lowering the extracellular $\mathrm{Ca}^{2+}$ concentration $\left(\left[\mathrm{Ca}^{2+}\right]_{\mathrm{e}}\right)$ to $10 \mathrm{nM}$ (Figure 4). Moreover, $\mathrm{HyNaC2} / 9 / 5$, which was insensitive to Hydra-RFamide I, was also opened by lowering $\left[\mathrm{Ca}^{2+}\right]_{\mathrm{e}}$, revealing that this combination of subunits assembles into a plasma membrane expressed ion channel, which was not gated by Hydra-RFamide I. In contrast, $\mathrm{HyNaC12}$, whether expressed alone or in combination with other HyNaCs, was not opened by lowering $\left[\mathrm{Ca}^{2+}\right]_{\mathrm{e}}$. Some DEG/ENaCs are constitutively opened by an amino acid substitution at the so-called DEG-position [38]. To further investigate whether $\mathrm{HyNaC12}$ forms a functional homomeric channel, we substituted a glycine at the DEGposition by threonine (G436T), which has a large side chain. This substitution did, however, not constitutively open $\mathrm{HyNaC12}$ (data not shown). This applies also for the co-expression of $\mathrm{HyNaC12}-\mathrm{G} 436 \mathrm{~T}$ together with pools of different wild-type $\mathrm{HyNaC}$ subunits, leaving the function of $\mathrm{HyNaC12}$ unknown.

Since Hydra-RFamide II had been shown previously to activate $\mathrm{HyNaC2} / 3 / 5$ [4], we tested Hydra-RFamides II to $\mathrm{V}$ for channel activation. All channels that were activated by Hydra-RFamide I were also activated by HydraRFamide II (data not shown). Instead Hydra-RFamide III to $\mathrm{V}(5 \mu \mathrm{M})$ failed to activate any functional $\mathrm{HyNaC}$ heterotrimer as well as $\mathrm{HyNaCs}$ containing $\mathrm{HyNaC12}$ (Figure 4).

\section{Expression pattern of $\mathrm{HyNaCs}$ reveals putative physiological subunit combinations}

The expression patterns of hynacs were studied by in situ hybridization (ISH). It had already been shown that hynac 2 to hynac 5 are expressed at the base of the tentacles of adult animals [3,4]. While hynac2 and hynac3 are uniformly expressed at the tentacle base, hynac4 and hynac 5 are asymmetrically expressed. hynac5 is strongly expressed at the oral side of each tentacle base with a gradient toward the aboral side [4], and hynac4 expression is restricted to the aboral side of the tentacle base [3]. Similar to hynac4 and hynac5, hynac6 and hynac11 were asymmetrically expressed at the base of the tentacles (Figures 5 and 6). While expression of hynac11 was restricted to the oral side, similar to hynac5, expression of hynac6 was restricted to the aboral side (Figure 6), similar to hynac4. These findings, together with the formation of 


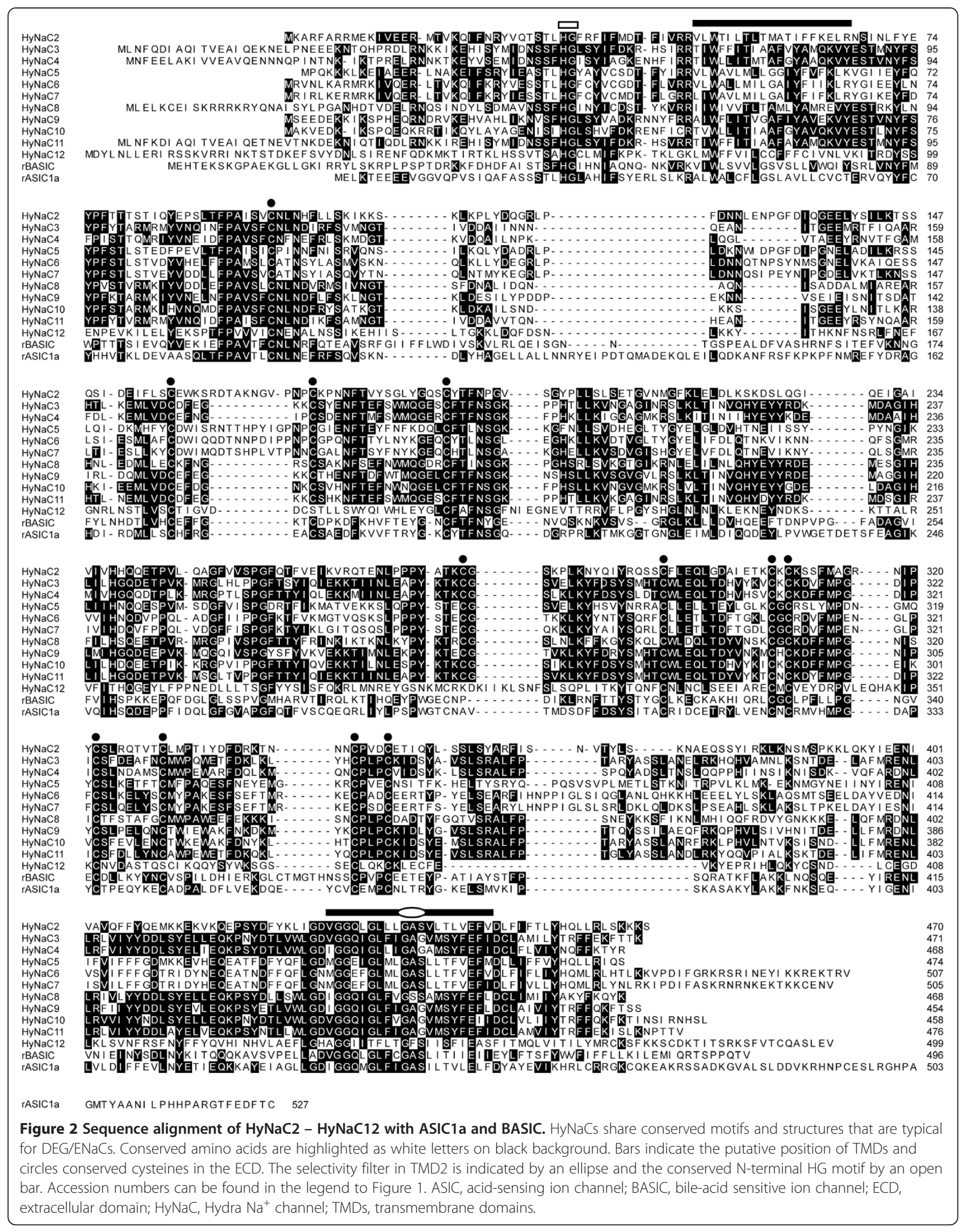




\begin{tabular}{|c|c|c|c|c|c|c|}
\hline 2 & 3 & 4 & 8 & 9 & 10 & 11 \\
\hline 5 & + & - & - & $(+)$ & - & + \\
\hline 6 & + & + & + & + & + & + \\
\hline 7 & + & + & - & + & + & + \\
\hline
\end{tabular}

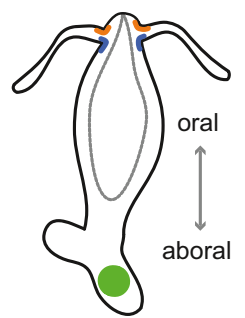

Figure 3 Rules of subunit assembly. Left, the table shows possible trimeric combinations of HyNaCs. HyNaC2 has to be present for a peptide-gated HyNaC. Subunits in the top row and the left row belong to the two subgroups defined by phylogenetic relation. A functional channel activated by Hydra-RFamides is represented by ' +', a non-functional channel by '-'. Activation by $\mathrm{Ca}^{2+}$ removal but insensitivity to Hydra-RFamides is indicated by '(+)'. Subunit combinations that are co-expressed in Hydra as revealed by ISH are shown on colored background. Orange represents expression at the oral side of the tentacle base, blue at the aboral side and green at the peduncle. Right, scheme illustrating expression sites of HyNaCs. HyNaC, Hydra $\mathrm{Na}^{+}$channel; ISH, in situ hybridization.

functional heteromeric channels in oocytes, suggest the presence of $\mathrm{HyNaC2} / 3 / 5$ and $\mathrm{HyNaC} 2 / 11 / 5$ heterotrimers at the oral side and of $\mathrm{HyNaC} 2 / 3 / 6$ and $\mathrm{HyNaC} 2 / 4 / 6$ heterotrimers at the aboral side of the tentacle base (Figure 3 ). The expression pattern is typical for expression in epitheliomuscular cells (for an example of hynac4 see Additional file 2: Figure S2).

The remaining hynacs were not expressed at the base of the tentacles (Figure 5). hynac7 and hynac10 were expressed at the peduncle (Figures 5 and 6), a region slightly above the basal disk. hynac10 was also expressed at the hypostome (oral region) (Figure 6). hynac8 and hynac9 were expressed over the whole body column but hynac 9 had an increased

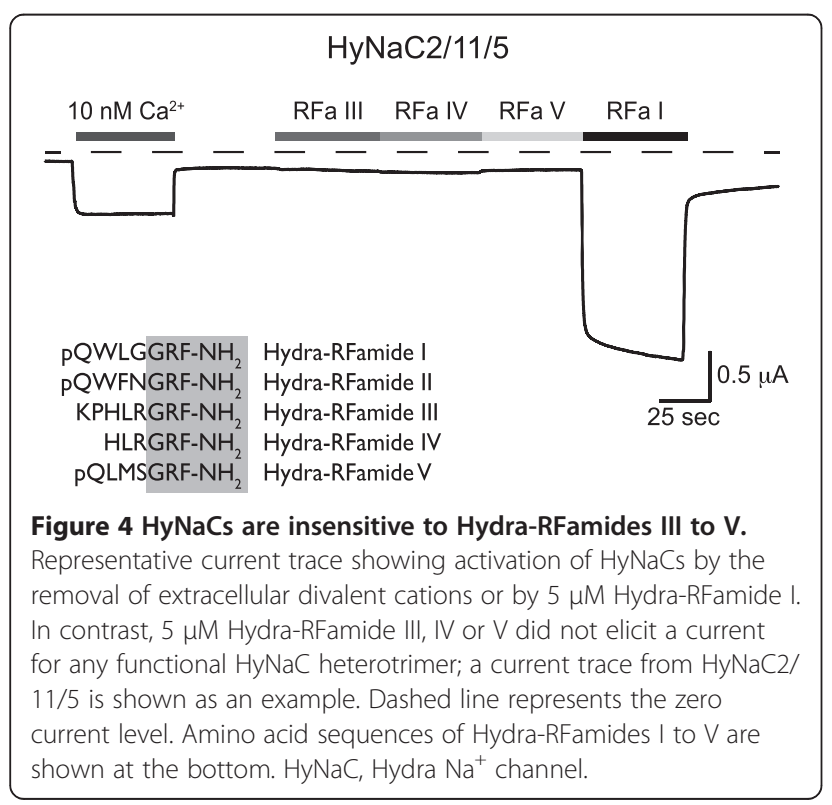

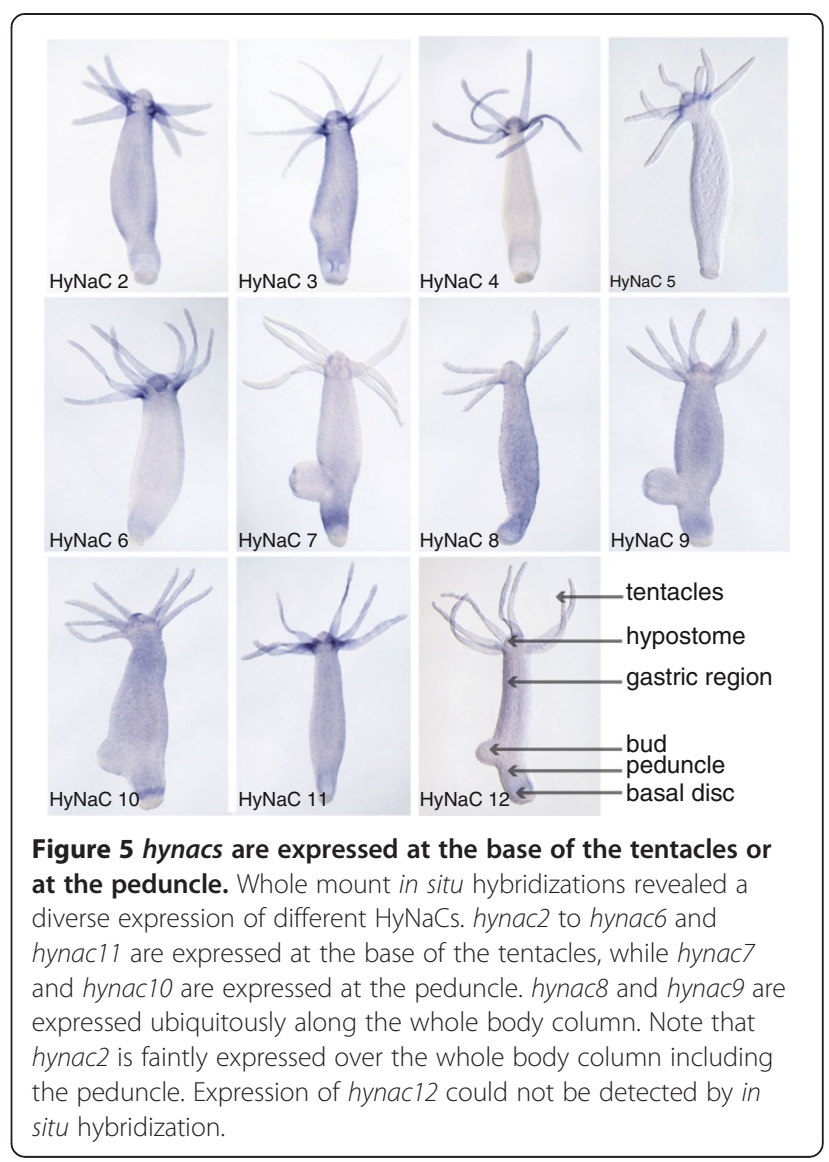

expression level in the peduncle region (Figure 5). Since $\mathrm{HyNaC2}$ was indispensable for formation of functional $\mathrm{HyNaCs}$ in the oocyte expression system, we re-evaluated its expression pattern and found slight expression of hynac 2 over the whole body column including the peduncle (Figure 5). These findings suggest the presence of $\mathrm{HyNaC} 2 / 9 / 7$ and $\mathrm{HyNaC} 2 / 10 / 7$ heterotrimers at the peduncle region. Since neurons expressing the preprohormone A gene that encodes the Hydra-RFamides I to IV are present at the tentacle bases, hypostome, upper gastric region and peduncle $[27,31,39]$, it is possible that cells expressing $\mathrm{HyNaCs}$ in the peduncle make synaptic junctions with neurons expressing Hydra-RFamides.

We were not able to detect hynac12 by in situ hybridization (Figure 5), suggesting that this subunit may be expressed in a restricted location or in low abundance. Moreover, hynac8 expression was faint and rather diffuse and we, therefore, could not assign a putative subunit combination containing $\mathrm{HyNaC8}$. Figure 3 summarizes the putative physiological subunit combinations of $\mathrm{HyNaCs}$.

\section{HyNaCs have high-affinity for Hydra-RFamide I and II}

We determined for HyNaCs with an overlapping expression pattern, and hence a putative occurrence in vivo, 


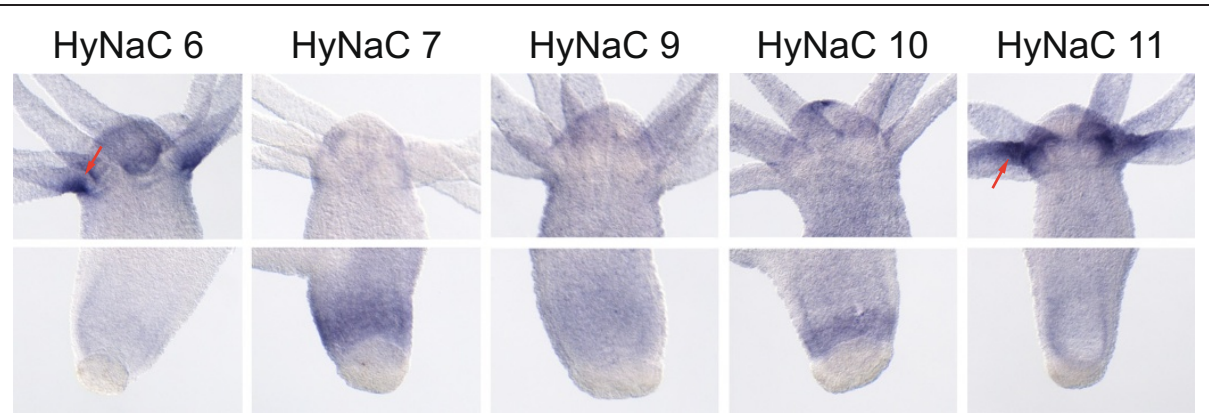

Figure 6 hynacs are differentially expressed at the base of the tentacles or at the peduncle. Magnifications of the in situ hybridizations for hynac6, hynac7 and hynac9 to hynac11 from Figure 5, demonstrating more precisely their expression patterns at the tentacle base and the peduncle.

apparent affinity for Hydra-RFamide I. HyNaC2/3/5 has a high $\mathrm{Ca}^{2+}$ permeability, which leads to activation of $\mathrm{Ca}^{2+}$-activated chloride channels (CaCCs) that are endogenously expressed in Xenopus oocytes [40]. A biphasic current with an initial peak and a slowly developing sustained phase is indicative for activation of CaCCs. All new $\mathrm{HyNaCs}$ had the same biphasic current as $\mathrm{HyNaC2} / 3 / 5$ (Figure $7 \mathrm{~B}$ ), suggesting high $\mathrm{Ca}^{2+}$ permeability. Therefore, we determined apparent affinity for Hydra-RFamide I with oocytes that had been injected with the $\mathrm{Ca}^{2+}$-chelator ethylene glycol tetraacetic acid (EGTA). In the presence of intracellular EGTA, Hydra-RFamide I induced simple onand off-responses, demonstrating that under these conditions currents were not strongly contaminated by currents through CaCCs. The $\mathrm{EC}_{50}$ for Hydra-RFamide I varied over more than two orders of magnitude from $0.04 \pm 0.01$
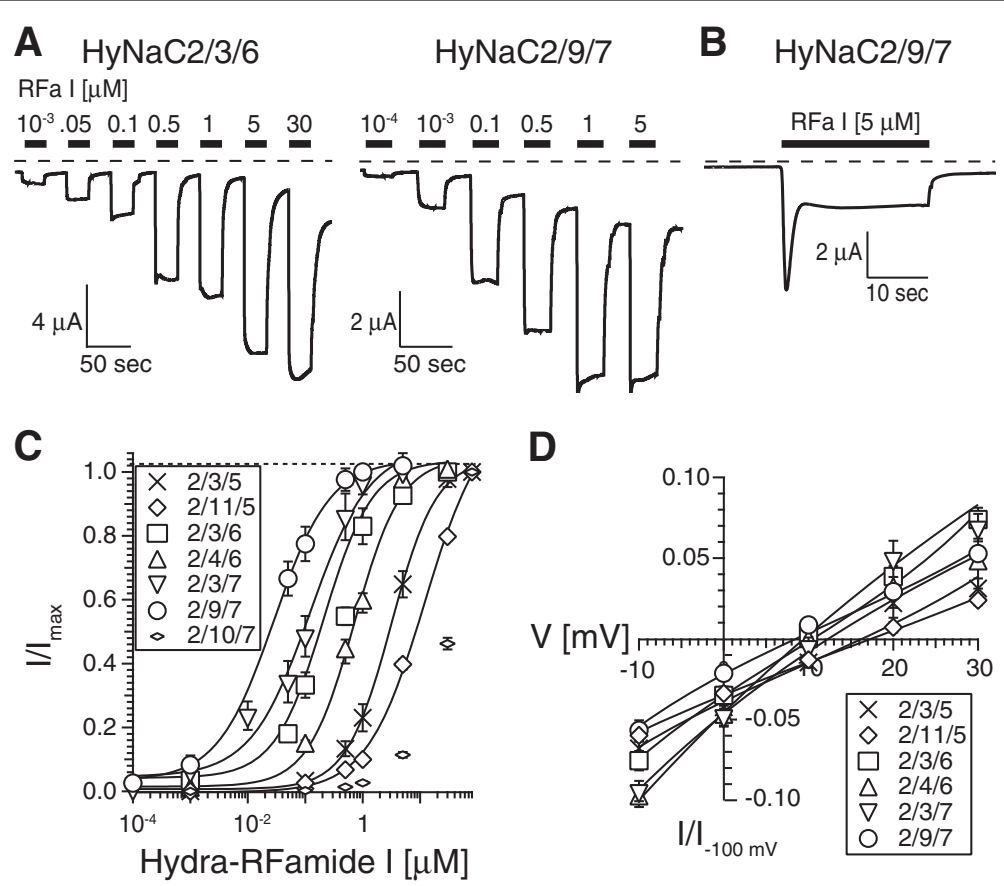

Figure 7 HyNaCs are activated by Hydra-RFamide I. A) Representative current traces showing concentration-dependent activation of HyNaC2/3/6 and HyNaC2/9/7 by Hydra-RFamide I when oocytes had been injected with EGTA. Dashed lines represent the zero current level. B) When oocytes had not been injected with EGTA, Hydra-RFamide I elicited biphasic currents; an oocyte expressing HyNaC2/9/7 is shown as an example. C) Concentration-response curves for $\mathrm{HyNaCs}$ and Hydra-RFamide I. Currents were normalized to the currents at the highest agonist concentration, which had amplitudes of $9.2 \pm 1.9 \mu \mathrm{A}(n=9 ; 2 / 3 / 5), 2.6 \pm 0.4 \mu \mathrm{A}(n=12 ; 2 / 11 / 5), 15.7 \pm 3.2 \mu \mathrm{A}(n=8 ; 2 / 3 / 6), 10.1 \pm 1.7 \mu \mathrm{A}(n=12$; $2 / 4 / 6), 19.6 \pm 1.8 \mu \mathrm{A}(n=10 ; 2 / 3 / 7), 7.2 \pm 0.9 \mu \mathrm{A}(n=10 ; 2 / 9 / 7)$ and $10.6 \pm 1.8 \mu \mathrm{A}(n=8 ; 2 / 10 / 7)$, respectively. Lines represent fits to the Hill equation. D) IN curves for putative physiological HyNaCs, revealing slightly positive reversal potentials. Voltage ramps were run from $-100 \mathrm{mV}$ to $+30 \mathrm{mV}$ in two seconds. Background currents had been subtracted by voltage ramps in the absence of agonist. Currents were normalized to the current at $-100 \mathrm{mV}$. EGTA, ethylene glycol tetraacetic acid; HyNaC, Hydra $\mathrm{Na}^{+}$channel. 
$\mu \mathrm{M}(\mathrm{n}=9)$ for $\mathrm{HyNaC2} / 9 / 7$ to $13.8 \pm 1.9 \mu \mathrm{M}(\mathrm{n}=12)$ for $\mathrm{HyNaC2} / 11 / 5$; for comparison, $\mathrm{HyNaC} 2 / 3 / 5$ has an $\mathrm{EC}_{50}$ of approximately $5 \mu \mathrm{M}$ [4]. For $\mathrm{HyNaC2} / 10 / 7$, the apparent affinity for Hydra-RFamide I was too low to calculate the $\mathrm{EC}_{50}$ precisely, since a saturating ligand concentration could not be reached (Figure $7 \mathrm{C}$, Table 1).

Previously, it had been shown that $\mathrm{HyNaC} 2 / 3 / 5$ has a ten times higher apparent affinity for Hydra-RFamide II than for Hydra-RFamide I [4]. We re-assessed apparent affinity of $\mathrm{HyNaC2} / 3 / 5$ for Hydra-RFamide II with EGTAinjected oocytes and found it to be $1.61 \pm 0.27 \mu \mathrm{M}(\mathrm{n}=14)$, slightly lower than reported for oocytes not injected with EGTA $(0.34 \pm 0.08 \mu \mathrm{M})$ [4]. Regarding the other putative physiological $\mathrm{HyNaC}$ heterotrimers, the $\mathrm{EC}_{50}$ for HydraRFamide II varied between $0.04 \pm 0.01 \mu \mathrm{M}(\mathrm{n}=8)$ for $\mathrm{HyNaC} 2 / 9 / 7$ and $0.95 \pm 0.16(\mathrm{n}=7)$ for $\mathrm{HyNaC} 2 / 11 / 5$ (Figure 8), which is in the same range as for HydraRFamide I. Only for $\mathrm{HyNaC} 2 / 11 / 5$ was the apparent affinity for Hydra-RFamide II about 15-fold higher than for Hydra-RFamide I (0.95 $\mu \mathrm{M}$ compared with $13.8 \mu \mathrm{M})$.

Similar to $\mathrm{HyNaC2/3/5} \mathrm{[4],} \mathrm{all} \mathrm{HyNaCs}$ had a slightly positive reversal potential $\mathrm{E}_{\mathrm{rev}}$ in standard bath $\left(\mathrm{E}_{\mathrm{rev}}\right.$ varied between $7.4 \pm 0.5 \mathrm{mV}, \mathrm{n}=10$, for $\mathrm{HyNaC} 2 / 9 / 7$ and $17.3 \pm$ $1.1 \mathrm{mV}, \mathrm{n}=8$, for $\mathrm{HyNaC2} / 11 / 5$; Figure 7D, Table 1 ), suggesting that they are unselective cation channels. HyNaC $2 / 3 / 5$ is characterized by a high $\mathrm{Ca}^{2+}$ permeability with a permeability ratio $\mathrm{P}_{\mathrm{Ca}} / \mathrm{P}_{\mathrm{Na}}=3.85$ [40]. We investigated the $\mathrm{Ca}^{2+}$ permeability of the new $\mathrm{HyNaCs}$ by replacing extracellular $\mathrm{Na}^{+}$by $\mathrm{Ca}^{2+}$. An increase in extracellular $\mathrm{Ca}^{2}$ + from 1 to $10 \mathrm{mM}$ resulted in a strong rightward shift of $\mathrm{E}_{\mathrm{rev}}$ (Figure 9), confirming $\mathrm{Ca}^{2+}$ permeability of $\mathrm{HyNaCs}$. The shift was in the same range as for $\mathrm{HyNaC2} / 3 / 5$ [40], suggesting a comparable $\mathrm{Ca}^{2+}$ permeability. We used $\mathrm{E}_{\mathrm{rev}}$ at $10 \mathrm{mM} \mathrm{Ca}^{2+}$ and at $140 \mathrm{mM} \mathrm{Na}^{+}$as the only permeant cations (see Methods) to calculate the relative permeability $\mathrm{P}_{\mathrm{Ca}} / \mathrm{P}_{\mathrm{Na}}$ for the new HyNaCs. $\mathrm{P}_{\mathrm{Ca}} / \mathrm{P}_{\mathrm{Na}}$ ranged from 2.66 for $\mathrm{HyNaC2} / 4 / 6(\mathrm{n}=9)$ to 3.72 for $\mathrm{HyNaC2}$ / $3 / 6(\mathrm{n}=9)$ (Table 1$)$, showing that all HyNaCs are highly $\mathrm{Ca}^{2+}$ permeable.

\section{Diminazene is a potent inhibitor of $\mathrm{HyNaCs}$ and inhibits the feeding reaction}

One of the hallmarks of DEG/ENaCs is the block by the diuretic amiloride [10]. Since the apparent amiloride affinity of $\mathrm{HyNaC} 2 / 3 / 5$ is quite low with an $\mathrm{IC}_{50}$ of approximately $120 \mu \mathrm{M}$ [4], amiloride is of limited utility for the study of HyNaCs. Recently, diminazene and related compounds have been reported to potently inhibit ASICs and BASIC but not ENaC $[41,42]$. Since ASICs and BASIC are closely related to $\mathrm{HyNaCs}$, we asked whether diminazene also inhibits HyNaCs. For most HyNaCs, $10 \mu \mathrm{M}$ of diminazene indeed almost completely blocked $\mathrm{HyNaC}$ currents. The $\mathrm{IC}_{50}$ for diminazene varied between $0.05 \pm 0.02 \mu \mathrm{M}$ $(\mathrm{n}=9)$ for $\mathrm{HyNaC} 2 / 4 / 6$ and $1.70 \pm 0.58 \mu \mathrm{M}(\mathrm{n}=9)$ for $\mathrm{HyNaC} 2 / 9 / 7$. Only HyNaC2/11/5 was blocked by diminazene with a low apparent affinity and an $\mathrm{IC}_{50}$ of $31.4 \pm 9.4$ $\mu \mathrm{M}$ ( $\mathrm{n}=8$ ). Although we did not determine apparent amiloride affinity for all $\mathrm{HyNaCs}$, based on a $20 \%$ to $70 \%$ inhibition of currents by $100 \mu \mathrm{M}$ amiloride (Figure 10, Table 1) one can estimate that diminazene has a $>100$-fold higher potency than amiloride for most $\mathrm{HyNaCs}$.

It has previously been shown that $100 \mu \mathrm{M}$ amiloride delays the feeding reaction of living Hydra [4], supporting the hypothesis that amiloride-sensitive channels are involved in the feeding reaction. To find further evidence that amiloride inhibited the feeding reaction via inhibition of $\mathrm{HyNaCs}$, we repeated these experiments with diminazene. Addition of glutathione (GSH; $10 \mu \mathrm{M}$ final concentration) induced tentacle contractions and animals moved the tentacles to their mouth. After about two minutes, $100 \%$ of animals had their tentacles completely curled. When animals were held in a medium containing $100 \mu \mathrm{M}$ diminazene, tentacle curling was slightly delayed. Increasing the concentration of diminazene to $200 \mu \mathrm{M}$, more strongly and significantly $(P \leq 0.01)$ delayed the initiation of tentacles movement such that tentacles were completely curled only after three minutes. When the concentration of diminazene was further increased to $300 \mu \mathrm{M}$, it strongly inhibited the feeding reaction such

Table 1 Summary of the biophysical properties of putatively physiological HyNaCs

\begin{tabular}{llllllll}
\hline HyNaC & $\mathbf{E C}_{\mathbf{5 0}}$ RFa I $[\boldsymbol{\mu M}]$ & Hill Coeff. & $\mathbf{I}_{\max } \mathrm{RFa} I[\boldsymbol{\mu A}]$ & $\mathbf{E}_{\mathbf{R e v}}[\mathbf{m V}]$ & $\mathbf{P}_{\mathbf{C a}} / \mathbf{P}_{\mathbf{N a}}$ & $\mathbf{I C}_{\mathbf{5 0}}$ Dimi $[\boldsymbol{\mu M}]$ & $\mathbf{I}_{\mathbf{A m i l}} / \mathbf{I}$ \\
\hline $\mathbf{2 / 3 / 5}$ & $3.48 \pm 0.55(9)$ & $1.2 \pm 0.1$ & $9.2 \pm 1.9(9)$ & $16 \pm 2(9)$ & 3.4 & $0.45 \pm 0.09(10)$ & $0.5 \pm 0.1(10)$ \\
$\mathbf{2 / 1 1 / 5}$ & $13.8 \pm 1.9(12)$ & $0.9 \pm 0.1$ & $2.7 \pm 0.3(12)$ & $17 \pm 1(8)$ & 3.3 & $31.4 \pm 9.4(8)$ & $0.7 \pm 0.1(8)$ \\
$\mathbf{2 / 3 / 6}$ & $0.29 \pm 0.06(10)$ & $1.2 \pm 0.2$ & $15.7 \pm 3.2(10)$ & $9 \pm 2(8)$ & 3.7 & $0.09 \pm 0.02(9)$ & $0.3 \pm 0.1(9)$ \\
$\mathbf{2 / 4 / 6}$ & $0.70 \pm 0.08(12)$ & $1.3 \pm 0.1$ & $8.8 \pm 1.5(12)$ & $14 \pm 3(9)$ & 2.7 & $0.05 \pm 0.02(9)$ & $0.3 \pm 0.1(9)$ \\
$\mathbf{2 / 3 / 7}$ & $0.19 \pm 0.04(14)$ & $1.6 \pm 0.4$ & $19.6 \pm 1.8(14)$ & $12 \pm 1(8)$ & 2.8 & $0.16 \pm 0.08(7)$ & $0.3 \pm 0.1(7)$ \\
$\mathbf{2 / 9 / 7}$ & $0.04 \pm 0.01(9)$ & $1.5 \pm 0.3$ & $7.4 \pm 0.9(11)$ & $7 \pm 1(10)$ & 2.9 & $1.70 \pm 0.58(9)$ & $0.3 \pm 0.1(9)$ \\
$\mathbf{2 / 1 0 / 7}$ & $>30(12)$ & n.d. & $11.9 \pm 1.5(12)$ & n.d. & n.d. & $5.40 \pm 1.45(6)$ & $0.8 \pm 0.1(6)$ \\
\hline
\end{tabular}

Properties are summarized for $\mathrm{HyNaCs}$ with a putative physiological role as judged by functionality in oocytes and by co-expression as revealed by ISH. $\mathrm{EC}_{50} \mathrm{RFa}_{\mathrm{I}}$, concentration of Hydra-RFamide I at which half-maximal activation was achieved; I $\max R$ RFa I, current amplitude at maximal concentration of Hydra-RFamide I; $E_{\text {Rev }}$ reversal potential of RFamide l-activated currents with standard bath; $\mathrm{P}_{\mathrm{Ca}} / \mathrm{P}_{\mathrm{Na}}$ relative permeability of $\mathrm{Ca}^{2+}$ to $\mathrm{Na}^{+} ; \mathrm{IC}_{50}$ Dimi, concentration of diminazene at which half-maximal inhibition was achieved; $I_{\text {Amil }} /$, relative current remaining after block by $100 \mu \mathrm{M}$ amiloride. Values indicate mean \pm standard error; the number $n$ of individual oocytes is indicated in brackets. $\mathrm{HyNaC}$, Hydra $\mathrm{Na}^{+}$channel; ISH, in situ hybridization. 


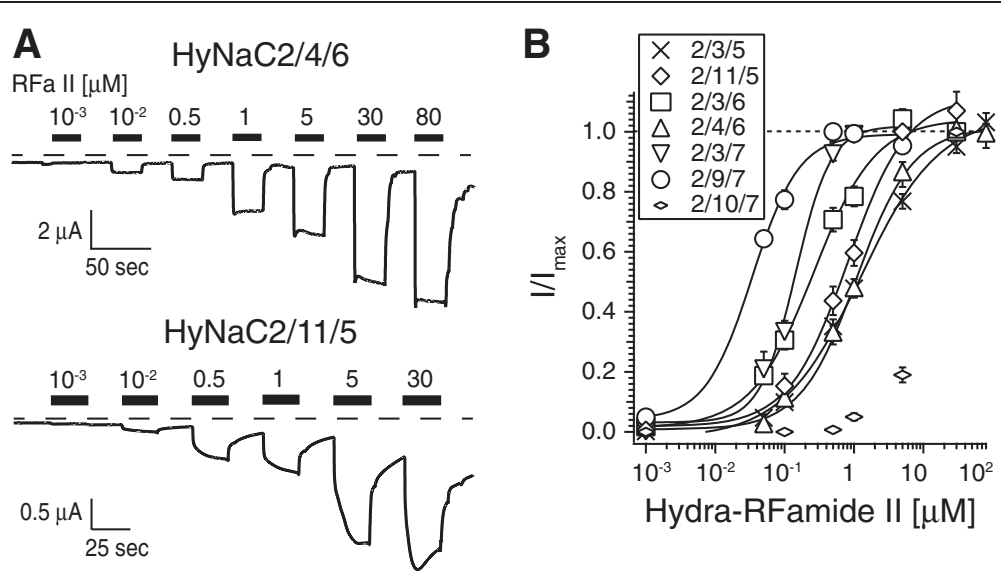

Figure $8 \mathrm{HyNaCs}$ are activated by Hydra-RFamide II. A) Representative current traces showing concentration-dependent activation of HyNaC2/4/6 and HyNaC2/11/5 by Hydra-RFamide II; oocytes had been injected with EGTA. Dashed lines represent the zero current level. B) Concentration-response curves for HyNaCs and Hydra-RFamide II. Currents were normalized to the currents at highest agonist concentration, which had amplitudes of $5.3 \pm 1.0 \mu \mathrm{A}(n=14 ; 2 / 3 / 5), 2.2 \pm 0.4 \mu \mathrm{A}(n=7 ; 2 / 11 / 5), 9.2 \pm 1.7 \mu \mathrm{A}(n=8 ; 2 / 3 / 6), 4.7 \pm 0.8 \mu \mathrm{A}(n=12 ; 2 / 4 / 6), 11.8 \pm 4.0$ $\mu \mathrm{A}(n=8 ; 2 / 3 / 7), 3.6 \pm 1.3 \mu \mathrm{A}(n=8 ; 2 / 9 / 7)$ and $1.1 \pm 0.4 \mu \mathrm{A}(n=7 ; 2 / 10 / 7)$, respectively. Lines represent fits to the Hill equation. EGTA, ethylene glycol tetraacetic acid; $\mathrm{HyNaC}, \mathrm{Hydra} \mathrm{Na}^{+}$channel.

that even after four minutes only $20 \%$ of the animals had their tentacles completely curled $(P \leq 0.001$; Figure 11$)$. Although the potency of diminazene in vivo was low, it should be taken into account that septate junctions provide a significant paracellular permeability barrier to the mesogleal space of Hydra [43]. Moreover, it appears that diminazene requires active transport mechanisms for cellular uptake [44,45], probably strongly limiting its transcellular transport; amiloride in contrast is, in part, uncharged at neutral $\mathrm{pH}$ and has a significant membrane permeability [46]. It is, therefore, conceivable that diminazene reached significantly lower concentrations in the mesogleal space of Hydra than in the bath. Therefore, these results support the idea that $\mathrm{HyNaCs}$ are involved in the feeding reaction of Hydra.

\section{Discussion}

\section{DEG/ENaCs in Hydra are peptide-gated}

In this study, we characterized the complete gene set of ten DEG/ENaC genes in the model cnidarian Hydra. We found genomic evidence for two further transcribed genes, that is, hynac1 and hynac12. Our data indicate,
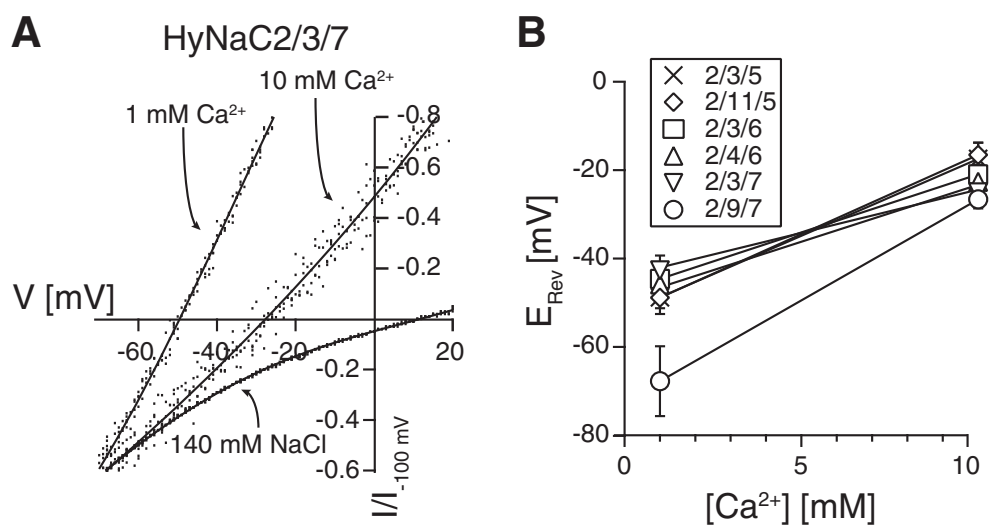

Figure 9 Change in extracellular $\mathrm{Ca}^{2+}$ concentration shifts the reversal potential of HyNaCs. A) Xenopus oocytes expressing HyNaC2/3/7 were activated by $100 \mathrm{nM}$ Hydra-RFamide I, corresponding to its $\mathrm{EC}_{50}$. The conductive cation in the extracellular solution was either $1 \mathrm{mM} \mathrm{Ca}^{2+}$ or $10 \mathrm{mM} \mathrm{Ca}^{2+}$, or $140 \mathrm{mM} \mathrm{Na}^{+}$. Reversal potentials were determined by voltage ramps from $-100 \mathrm{mV}$ to $+30 \mathrm{mV}$ in two seconds. Background currents had been subtracted by voltage ramps in the absence of agonist. B) Diagram showing the shift of the reversal potentials for different $\mathrm{HyNaC}$ combinations, when the extracellular solution contained 1 or $10 \mathrm{mM} \mathrm{Ca}{ }^{2+}$ as the only conductive ion. HyNaCs had been activated by a concentration of Hydra-RFamide I that corresponds to the individual $\mathrm{EC}_{50}$. $\mathrm{HyNaCs}$, Hydra $\mathrm{Na}^{+}$channels. 


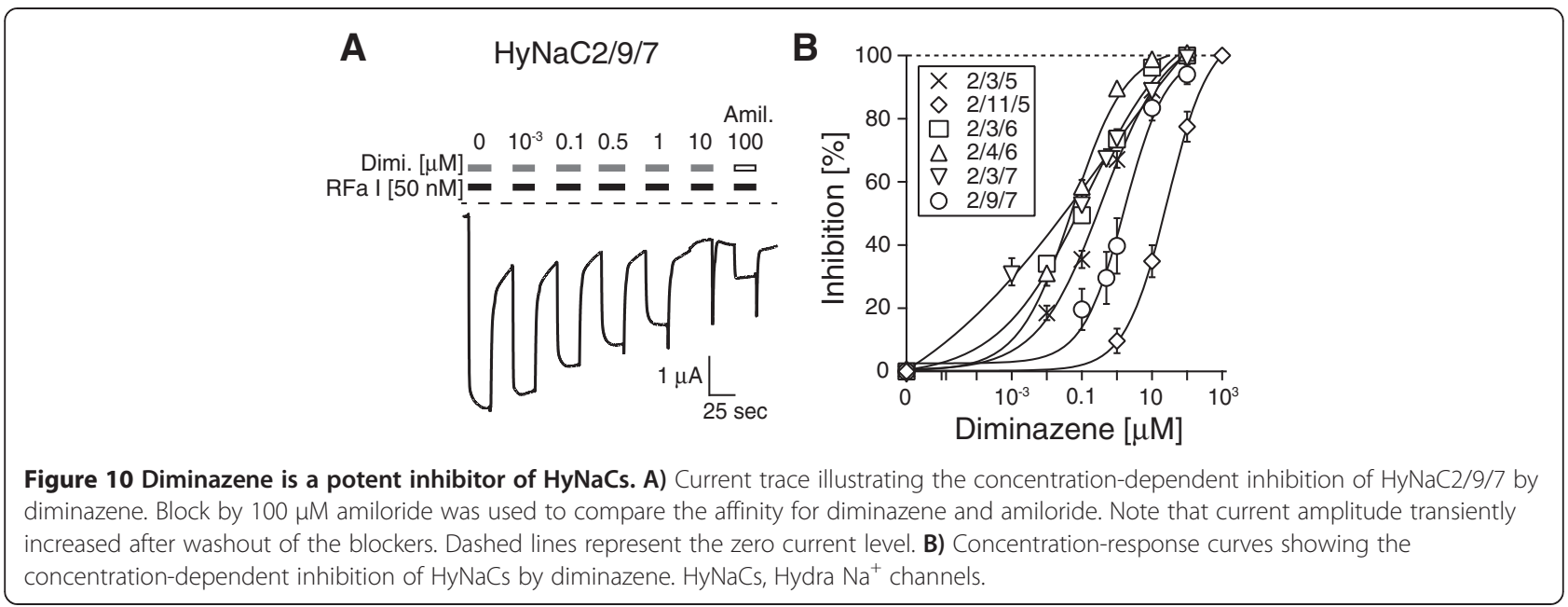

however, that these are pseudogenes that probably arose by retrotransposition from a common precursor with other $\mathrm{HyNaCs}$ [32]. The remaining ten genes give rise to ten $\mathrm{HyNaC}$ subunits that assemble in a variety of combinations into heteromeric ion channels.

$\mathrm{HyNaCs}$ are more closely related to each other than to any other $\mathrm{DEG} / \mathrm{ENaC}$, forming a sub-branch on the phylogenetic tree in Figure 1. Closest relatives are ASICs [13] and BASIC [14]. HyNaCs form two lower order subgroups within the $\mathrm{HyNaC}$ branch (Figure 1). Subgroup 1 (dark blue in Figure 1) contains HyNaC2, HyNaC5, HyNaC6 and $\mathrm{HyNaC7}$, while subgroup 2 (light blue in Figure 1) contains $\mathrm{HyNaC}, \mathrm{HyNaC4}$ and $\mathrm{HyNaC} 8$ to $\mathrm{HyNaC11}$. Combinatorial expression of individual $\mathrm{HyNaCs}$ revealed the rules for subunit assembly. $\mathrm{HyNaC2}$ was present in all functional

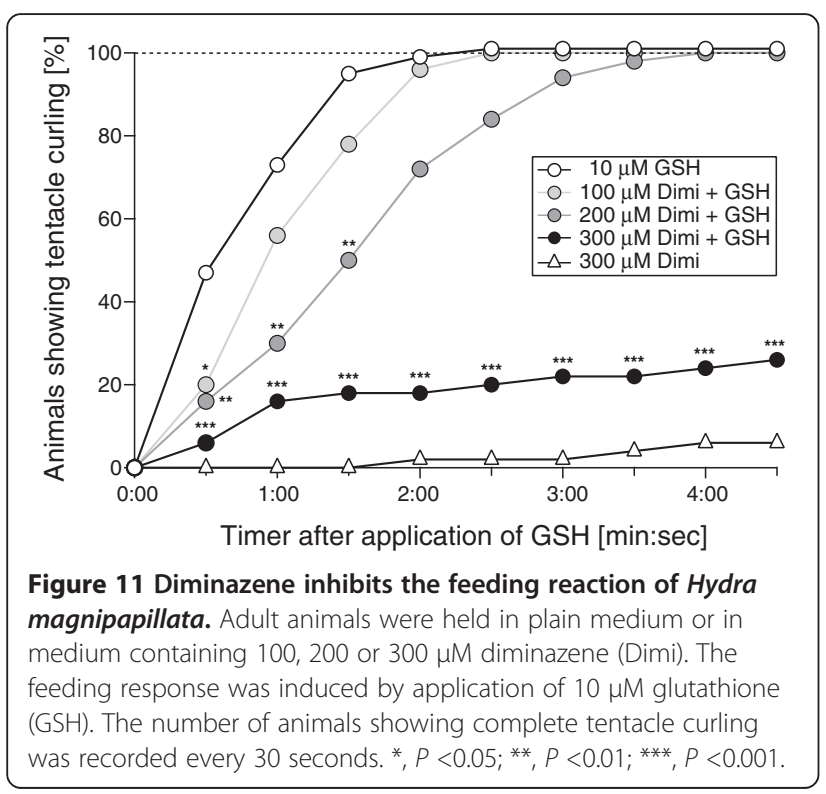

channels. The second subunit had to be from subgroup 2 and the third was another subunit from subgroup 1, closely related to $\mathrm{HyNaC} 2 . \mathrm{HyNaC} 2 / 3$ is the only $\mathrm{HyNaC}$ that contains only two different subunits yet is functional [3]. The presence of a HyNaC2-like subunit in all other channels makes $2 / 2 / 3$ (and not $2 / 3 / 3$ ) the likely composition of $\mathrm{HyNaC} 2 / 3$.

According to the rules described above, HyNaCs could theoretically assemble in 18 different combinations (Figure 3). We found that 13 of these combinations are activated by Hydra-RFamides I and II (Figure 3). One other combination $(\mathrm{HyNaC2} / 9 / 5)$ was not activated by Hydra-RFamides I and II but by removing extracellular divalent cations, indicating correctly assembled plasma membrane expressed channels. It is therefore possible that $\mathrm{HyNaC2} / 9 / 5$ is activated by another neuropeptide than RFamides. Since the other four theoretical subunit combinations could neither be activated by RFamide neuropeptides nor by removal of divalent cations, it is likely that these combinations do not assemble in functional ion channels; at least not in the oocyte expression system. With the exception of $\mathrm{HyNaC} 2 / 10 / 7$, the functional peptide-activated channels all had high apparent ligand affinity; for some, such as $\mathrm{HyNaC2} / 9 / 7$, nanomolar concentrations of Hydra-RFamides I and II elicited robust currents (Figure 7). The high affinity for RFamides I and II renders it highly unlikely that other neuropeptides also activate $\mathrm{HyNaCs}$ with similar affinity. We, therefore, conclude that the physiological ligands of HyNaCs are Hydra-RFamides I and II, with the possible exceptions of $\mathrm{HyNaC2} / 9 / 5$ and $\mathrm{HyNaC} 2 / 10 / 7$. This conclusion lends support to the hypothesis that the common ancestor of DEG/ENaCs was a peptide-gated channel. A definite answer to this question, however, requires the analysis of $\mathrm{DEG} / \mathrm{ENaCs}$ from more nonbilaterian animals. 


\section{HyNaCs may contribute to neuromuscular transmission in Hydra}

For six of the thirteen heterotrimers, which are activated by RFamides in the oocyte expression system, the three subunits are co-expressed (Figure 5). It is therefore likely that these six heterotrimers form peptide-gated channels in situ. Due to the limited sensitivity of ISH, we cannot exclude that further combinations exist in situ. In particular, we could not assign the locus of expression of $\mathrm{HyNaC8}$. In summary, our results suggest that two different $\mathrm{HyNaCs}$ are present at the oral $(\mathrm{HyNaC} 2 / 3 / 5$ and $\mathrm{HyNaC2} / 11 / 5)$ and two at the aboral side of the tentacle base $(\mathrm{HyNaC} 2 / 3 / 6$ and $\mathrm{HyNaC} 2 / 4 / 6)$, respectively. Two other $\mathrm{HyNaCs}$ are expressed at the peduncle $(\mathrm{HyNaC2} /$ 9/7 and $\mathrm{HyNaC2} / 10 / 7)$. Since these channels are all activated by Hydra-RFamides I and II, this variety is unexpected. We speculate that the variety of HyNaCs helps to fine tune ligand affinity and perhaps subcellular location of RFamide-gated channels.

What is the function of $\mathrm{HyNaCs}$ ? ISH from this and previous $[3,4]$ studies suggests that $\mathrm{HyNaCs}$ are expressed in epitheliomuscular cells, which function as muscle cells. Since Hydra-RFamides are contained within large dense core vesicles in axon terminals of neurons contacting epitheliomuscular cells [28,29], our results identify $\mathrm{HyNaCs}$ as a candidate post-synaptic receptor for Hydra-RFamides, which could depolarize muscle cells to induce contractions. In agreement with this speculation, amiloride [4] and diminazene, blockers of $\mathrm{HyNaCs}$, significantly delayed the feeding reaction of Hydra (Figure 11). In vertebrates and arthropods, however, neuromuscular transmission uses the small molecule transmitters, acetylcholine and glutamate, respectively. Sequencing of the Hydra genome indeed revealed seventeen genes coding for nicotinic acetylcholine receptor subunits but other key components of the vertebrate neuromuscular junction are not present in Hydra and the authors concluded that a canonical bilaterian neuromuscular junction was not present in the last common ancestor of cnidarians and bilaterians [32]. Moreover, it has recently been suggested that striated muscles evolved convergently in cnidarians and bilaterians from cells with ancient contractile machinery [47]. Assuming that the junction with neurons of striated muscle cells evolved after or at the same time as the evolution of striated muscles, this finding would explain why cnidarian and bilaterian muscles might use different transmitters at their junction with neurons. In summary, our results suggest that HydraRFamides I and II and HyNaCs should be considered as a transmitter-receptor pair mediating fast neuromuscular transmission in Hydra.

One feature of $\mathrm{HyNaCs}$ that is unique for DEG/ENaCs is their high $\mathrm{Ca}^{2+}$ permeability. If $\mathrm{HyNaCs}$ indeed carried an excitatory post-synaptic current in muscle cells then $\mathrm{Ca}^{2+}$ flux through $\mathrm{HyNaCs}$ could directly induce muscle contractions independent of release from intracellular stores. A high $\mathrm{Ca}^{2+}$ permeability might also confer an advantage in a freshwater animal like Hydra with a low extracellular $\mathrm{Na}^{+}$concentration. In Hydra littoralis (a member of the $H$. vulgaris group, [48]) an extracellular osmolality of $16.8 \mathrm{mM} / \mathrm{kg}$ has been measured [49], significantly higher than in the surrounding freshwater but much lower than in most other animals. Thus, $\mathrm{Ca}^{2+}$ may carry a significant fraction of the excitatory current in the freshwater animal Hydra.

\section{Conclusions}

Our study identifies in Hydra a variety of ion channels gated by Hydra-RFamide neuropeptides. Their expression in epithelial cells at the base of the tentacles and in the peduncle suggests a role for these channels in neuromuscular transmission. Thus, our results support the hypothesis that Hydra uses neuropeptides for fast neuromuscular transmission.

\section{Methods \\ Cloning of new HyNaCs}

Using the DNA sequences of $\mathrm{HyNaC} 2$ to $\mathrm{HyNaC5}$, we performed a BLAST search against the genomic database of Hydra magnipapillata at the National Center for Biotechnology Information and identified the full-length protein sequence for three new $\mathrm{HyNaCs}$; these $\mathrm{HyNaCs}$ were later named $\mathrm{HyNaC6}, \mathrm{HyNaC} 9$ and $\mathrm{HyNaC10}$, respectively. The complete coding sequences of these three $\mathrm{HyNaCs}$ were directly amplified by PCR with specific primers using cDNA extracted from adult one-day starved budding stage animals of Hydra magnipapillata strain 105. Other genomic sequences with homology to $\mathrm{HyNaCs}$ were used to design primers for 3 ' - and 5'-RACE. Sequences obtained by RACE-PCRs were assembled to full-length sequences, which were then amplified by PCR with specific primers using Hydra cDNA. The new HyNaC sequences were submitted to the EMBL-EBI-database with accession numbers HyNaC6 [EMBL:HG422725], HyNaC7 [EMBL: HG422726], HyNaC8 [EMBL:HG422727], HyNaC9 [EMBL: HG422728], HyNaC10 [EMBL:HG422729], HyNaC11 [EMBL: HG422730], HyNaC12 [EMBL:HG422731].

\section{Analysis of phylogenetic relationship of $\mathrm{HyNaCs}$}

To analyze the phyologenetic relationship of $\mathrm{HyNaC}$ subunits, their sequences were aligned with the sequences of other DEG/ENaC channels using ClustalX (v.2.1); highly divergent sequences at the $\mathrm{N}$ - and $\mathrm{C}$ terminus as well as in the extracellular loop were deleted to improve the alignment by automated 1 method of trimAl (v.1.3). The best model for amino acid substitution was determined with ProtTest 3 [50]. Phylogenetic trees were established by neighbor-joining analysis with ClustalX (v.2.1), maximum likelihood analysis with PhyML 
(v.3.0) [51] and Bayesian analysis with MrBayes (v.3.2). For maximum likelihood analysis we used the WAG model of protein evolution and the NNI tree topology search option; bootstrap support values were obtained from 100 bootstrap samples. During Bayesian analysis an average standard deviation $\leq 0.0001$ was estimated. Therefore we ran $10^{7}$ generations with one chain without Metropolis coupling. After a burn-in of $10^{5}$ generations every 1,000th tree was sampled. Regarding the phylogenetic relations of $\mathrm{HyNaCs}$, ASICs and BASICs, all three phylogenetic analyses revealed comparable results. Relations of other DEG/ENaCs were slightly different. A ClustalW analysis of all $\mathrm{HyNaC}$ subunits, rASIC1a, and rBASIC was performed with the software DNASTAR (v.10.0.1; Madison, WI, USA) to draw the alignment of Figure 2.

\section{In situ hybridization}

Expression patterns of $\mathrm{HyNaCs}$ were analyzed by whole mount in situ hybridization [52]. For all new $\mathrm{HyNaCs}$, probes for in situ hybridization were subcloned into pBluescript KS; they had lengths between 600 and 1,400 $\mathrm{bp}$, depending on the staining efficiency. Probes were detected with an antibody conjugated to an alkaline phosphatase and BMP Purple as substrate.

\section{Electrophysiology}

To study the biophysical properties of the new HyNaCs, cRNA of $\mathrm{HyNaCs}$ was synthesized in vitro (mMessage mMachine kit, Ambion, Austin, Texas, USA) and injected into Xenopus laevis oocytes of stage V and VI. After injection, oocytes were incubated for one to two days at $19^{\circ} \mathrm{C}$ in oocyte Ringer's solution 2 (OR-2), which contained (in mM): $82.5 \mathrm{NaCl}, 2.5 \mathrm{KCl}, 1.0 \mathrm{Na}_{2} \mathrm{HPO}_{4}$, 1.0 $\mathrm{MgCl}_{2}, 1.0 \mathrm{CaCl}_{2}$, 5.0 HEPES, $0.5 \mathrm{~g} / \mathrm{l} \mathrm{PVP,} \mathrm{1,000} \mathrm{U/l}$ penicillin and $10 \mathrm{mg} / \mathrm{l}$ streptomycin, $\mathrm{NaOH}$ was used to adjust $\mathrm{pH}$ to 7.3. Whole cell currents were recorded from defolliculated oocytes by two-electrode voltageclamp (TEVC) using the amplifier TurboTec 03X (npi electronic GmbH, Tamm, Germany). Standard bath solution (in mM: $140 \mathrm{NaCl}, 10 \mathrm{HEPES}, 1.8 \mathrm{CaCl}_{2}$ and 1.0 $\mathrm{MgCl}_{2} \cdot \mathrm{NaOH}$ was used to adjust $\mathrm{pH}$ to 7.4.) was exchanged by a pump driven system combined with the oocyte testing carousel (OTC), which was controlled by the interface OTC-20 [53]. To control the OTC-20 and to record the data to the hard drive, the software CellWorks (version 5.1.1; npi electronic GmbH, Tamm, Germany) was used. Data were sampled at 0.1 to $1 \mathrm{kHz}$ and filtered at $20 \mathrm{~Hz}$. HyNaCs are permeable for $\mathrm{Ca}^{2+}$, activating an endogenous $\mathrm{CaCC}$ [40]. Therefore, unless otherwise indicated, oocytes were injected with $50 \mathrm{nl}$ of 20 mM EGTA 30 to 180 minutes before measurements. Reversal potentials and $\mathrm{Ca}^{2+}$ permeabilities of $\mathrm{HyNaCs}$ were studied as described previously [40].

\section{Data analysis}

$\mathrm{EC}_{50}$ values for Hydra-RFamides I and II as well as $\mathrm{IC}_{50}$ values for diminazene were determined by fits to the Hill equation using IGOR Pro (version 6.06, WaveMetrics, Inc.). Reversal potentials were defined as the first recorded data point, where currents reversed signs from negative to positive. For I/V relationships, voltage ramps in the absence of the agonist were used to subtract background conductances. Results are reported as mean \pm standard error.

\section{Additional files}

Additional file 1: Figure S1. Phylogenetic tree for the DEG/ENaC family generated by maximum likelihood analysis. The phylogenetic tree was generated by maximum likelihood analysis with PhyML (see Methods). Support values are indicated. Scale bar indicates amino acid exchanges per site. Color code, abbrevations of species names, and accession numbers are as in Figure 1.

Additional file 2: Figure S2. High magnification of whole mount in situ hybridization for hynac4 revealing expression in epitheliomuscular cells at the base of the tentacles.

\section{Abbreviations}

ASIC: acid-sensing ion channel; BASIC: bile acid-sensitive ion channel; CaCCs: $\mathrm{Ca}^{+}$-activated chloride channels; DEG: degenerin; ECD: extracellular domain; EGTA: ethylene glycol tetraacetic acid; ENaC: epithelial $\mathrm{Na}^{+}$channel; FaNaC: FMRFamide-gated $\mathrm{Na}^{+}$channel; HyNaC: Hydra $\mathrm{Na}^{+}$channel; ISH: in situ hybridization; PPK: pickpocket; TMD: transmembrane domain.

\section{Competing interests}

The authors declare that they have no competing interests.

\section{Authors' contributions}

MA and SD carried out the molecular cloning of new HyNaC subunits. MA made the phylogenetic analyses, characterized HyNaCs electrophysiologically in Xenopus oocytes, performed the statistical analysis and participated in drafting the manuscript. AK carried out the in situ hybridizations and the pharmacological studies with live Hydra. TWH and SG conceived the study, and participated in its design and coordination and drafted the manuscript. All authors read and approved the final manuscript.

\section{Acknowledgements}

We thank S. Lenz for expert technical assistance and Y. Moran (Hebrew University, Jerusalem) for advice on phylogenetic analysis. This project was supported by grants \# GR 1771/7-1 and HO 1088/7-1 from the Deutsche Forschungsgemeinschaft to SG and $\mathrm{TWH}$, respectively.

\section{Author details}

'Department of Physiology, RWTH Aachen University, D-52074 Aachen, Germany. ${ }^{2}$ Molecular Evolution and Genomics, Center for Organismal Studies, D-69120 Heidelberg, Germany.

Received: 9 July 2014 Accepted: 3 October 2014

Published online: 14 October 2014

\section{References}

1. Cottrell GA, Green KA, Davies NW: The neuropeptide Phe-Met-Arg-Phe$\mathrm{NH} 2$ (FMRFamide) can activate a ligand-gated ion channel in Helix neurones. Pflugers Arch 1990, 416:612-614.

2. Lingueglia $E$, Champigny G, Lazdunski M, Barbry P: Cloning of the amiloride-sensitive FMRFamide peptide-gated sodium channel. Nature 1995, 378:730-733.

3. Golubovic A, Kuhn A, Williamson M, Kalbacher H, Holstein TW, Grimmelikhuijzen CJ, Gründer S: A peptide-gated ion channel from the freshwater polyp Hydra. J Biol Chem 2007, 282:35098-35103. 
4. Dürrnagel $S$, Kuhn A, Tsiairis CD, Williamson M, Kalbacher $H$, Grimmelikhuijzen CJ, Holstein TW, Gründer S: Three homologous subunits form a high affinity peptide-gated ion channel in Hydra. J Biol Chem 2010, 285:11958-11965.

5. Jékely G: Global view of the evolution and diversity of metazoan neuropeptide signaling. Proc Natl Acad Sci U S A 2013, 110:8702-8707.

6. Green KA, Falconer SW, Cottrell GA: The neuropeptide Phe-Met-Arg-Phe$\mathrm{NH} 2$ (FMRFamide) directly gates two ion channels in an identified Helix neurone. Pflugers Arch 1994, 428:232-240.

7. Davey F, Harris SJ, Cottrell GA: Histochemical localisation of FMRFamidegated $\mathrm{Na}+$ channels in Helisoma triolvis and Helix aspersa neurones. J Neurocytol 2001, 30:877-884.

8. Perry SJ, Straub VA, Schofield MG, Burke JF, Benjamin PR: Neuronal expression of an FMRFamide-gated $\mathrm{Na}+$ channel and its modulation by acid pH. J Neurosci 2001, 21:5559-5567.

9. Walker RJ, Papaioannou S, Holden-Dye L: A review of FMRFamide- and RFamide-like peptides in metazoa. Invert Neurosci 2009, 9:111-153.

10. Kellenberger S, Schild L: Epithelial sodium channel/degenerin family of ion channels: a variety of functions for a shared structure. Physio/ Rev 2002, 82:735-767.

11. O'Hagan R, Chalfie M, Goodman MB: The MEC-4 DEG/ENaC channel of caenorhabditis elegans touch receptor neurons transduces mechanical signals. Nat Neurosci 2005, 8:43-50.

12. Canessa CM, Schild L, Buell G, Thorens B, Gautschi I, Horisberger JD, Rossier BC: Amiloride-sensitive epithelial $\mathrm{Na}+$ channel is made of three homologous subunits. Nature 1994, 367:463-467.

13. Waldmann R, Champigny G, Bassilana F, Heurteaux C, Lazdunski M: A proton-gated cation channel involved in acid-sensing. Nature 1997, 386:173-177.

14. Wiemuth D, Sahin H, Falkenburger BH, Lefevre CM, Wasmuth HE, Gründer S: BASIC-a bile acid-sensitive ion channel highly expressed in bile ducts. FASEB J 2012, 26:4122-4130.

15. Askwith CC, Cheng C, Ikuma M, Benson C, Price MP, Welsh MJ: Neuropeptide FF and FMRFamide potentiate acid-evoked currents from sensory neurons and proton-gated DEG/ENaC channels. Neuron 2000, 26:133-141.

16. Liu L, Leonard AS, Motto DG, Feller MA, Price MP, Johnson WA, Welsh MJ: Contribution of drosophila DEG/ENaC genes to salt taste. Neuron 2003, 39:133-146.

17. Cameron P, Hiroi M, Ngai J, Scott K: The molecular basis for water taste in Drosophila. Nature 2010, 465:91-95.

18. Chen Z, Wang Q, Wang Z: The amiloride-sensitive epithelial $\mathrm{Na}+$ channel PPK28 is essential for drosophila gustatory water reception. J Neurosci 2010, 30:6247-6252.

19. Zhong $L$, Hwang RY, Tracey WD: Pickpocket is a DEG/ENaC protein required for mechanical nociception in Drosophila larvae. Curr Biol 2010 20:429-434.

20. Starostina E, Liu T, Vijayan V, Zheng Z, Siwicki KK, Pikielny CW: A Drosophila DEG/ENaC subunit functions specifically in gustatory neurons required for male courtship behavior. J Neurosci 2012, 32:4665-4674.

21. Thistle R, Cameron P, Ghorayshi A, Dennison L, Scott K: Contact chemoreceptors mediate male-male repulsion and male-female attraction during Drosophila courtship. Cell 2012, 149:1140-1151.

22. Liu T, Starostina E, Vijayan V, Pikielny CW: Two Drosophila DEG/ENaC channel subunits have distinct functions in gustatory neurons that activate male courtship. J Neurosci 2012, 32:11879-11889.

23. Jasti J, Furukawa H, Gonzales EB, Gouaux E: Structure of acid-sensing ion channel 1 at 1.9 a resolution and low pH. Nature 2007, 449:316-323.

24. Gonzales EB, Kawate T, Gouaux E: Pore architecture and ion sites in acid-sensing ion channels and P2X receptors. Nature 2009, 460:599-604.

25. Bartoi T, Augustinowski K, Polleichtner G, Gründer S, Ulbrich MH: Acid-sensing ion channel (ASIC) $1 \mathrm{a} / 2 \mathrm{a}$ heteromers have a flexible 2:1/1:2 stoichiometry. Proc Natl Acad Sci U S A 2014, 111:8281-8286.

26. Moosler A, Rinehart KL, Grimmelikhuijzen CJ: Isolation of four novel neuropeptides, the hydra-RFamides I-IV, from Hydra magnipapillata. Biochem Biophys Res Commun 1996, 229:596-602.

27. Darmer D, Hauser F, Nothacker HP, Bosch TC, Williamson M Grimmelikhuijzen CJ: Three different prohormones yield a variety of Hydra-RFamide (Arg-Phe-NH2) neuropeptides in Hydra magnipapillata. Biochem J 1998, 332:403-412
28. Koizumi O, Wilson JD, Grimmelikhuijzen CJ, Westfall JA: Ultrastructural localization of RFamide-like peptides in neuronal dense-cored vesicles in the peduncle of Hydra. J Exp Zool 1989, 249:17-22.

29. Westfall JA: Ultrastructure of synapses in the first-evolved nervous systems. J Neurocytol 1996, 25:735-746.

30. Grimmelikhuijzen CJ, Leviev I, Carstensen K: Peptides in the nervous systems of cnidarians: structure, function, and biosynthesis. Int Rev Cytol 1996, 167:37-89.

31. Hansen GN, Williamson M, Grimmelikhuijzen CJ: Two-color double-labeling in situ hybridization of whole-mount Hydra using RNA probes for five different Hydra neuropeptide preprohormones: evidence for colocalization. Cell Tissue Res 2000, 301:245-253.

32. Chapman JA, Kirkness EF, Simakov O, Hampson SE, Mitros T, Weinmaier T, Rattei T, Balasubramanian PG, Borman J, Busam D, Disbennett K, Pfannkoch C, Sumin N, Sutton GG, Viswanathan LD, Walenz B, Goodstein DM, Hellsten U, Kawashima T, Prochnik SE, Putnam NH, Shu S, Blumberg B, Dana CE, Gee L, Kibler DF, Law L, Lindgens D, Martinez DE, Peng J, et al: The dynamic genome of Hydra. Nature 2010, 464:592-596.

33. Gründer S, Jaeger NF, Gautschi I, Schild L, Rossier BC: Identification of a highly conserved sequence at the $\mathrm{N}$-terminus of the epithelial $\mathrm{Na}+$ channel alpha subunit involved in gating. Pflugers Arch 1999, 438:709-715.

34. Kellenberger S, Gautschi I, Schild L: A single point mutation in the pore region of the epithelial $\mathrm{Na}+$ channel changes ion selectivity by modifying molecular sieving. Proc Natl Acad Sci U S A 1999, 96:4170-4175.

35. Snyder PM, Olson DR, Bucher DB: A pore segment in DEG/ENaC $\mathrm{Na}(+)$ channels. J Biol Chem 1999, 274:28484-28490.

36. Firsov D, Robert-Nicoud M, Gründer S, Schild L, Rossier BC: Mutational analysis of cysteine-rich domains of the epithelium sodium channel (ENaC). Identification of cysteines essential for channel expression at the cell surface. J Biol Chem 1999, 274:2743-2749.

37. Wiemuth $D$, Gründer S: A single amino acid tunes Ca2+ inhibition of brain liver intestine $\mathrm{Na}+$ channel (BLINaC). J Biol Chem 2010, 285:30404-30410.

38. Driscoll $M$, Chalfie M: The mec- 4 gene is a member of a family of Caenorhabditis elegans genes that can mutate to induce neuronal degeneration. Nature 1991, 349:588-593.

39. Grimmelikhuijzen CJ: Antisera to the sequence Arg-Phe-amide visualize neuronal centralization in hydroid polyps. Cell Tissue Res 1985, 241:171-182.

40. Dürrnagel $\mathrm{S}$, Falkenburger $\mathrm{BH}$, Gründer $\mathrm{S}$ : High $\mathrm{Ca}(2+)$ permeability of a peptide-gated DEG/ENaC from Hydra. J Gen Physio/ 2012, 140:391-402.

41. Chen X, Qiu L, Li M, Dürrnagel S, Orser BA, Xiong ZG, MacDonald JF: Diarylamidines: high potency inhibitors of acid-sensing ion channels. Neuropharmacology 2010, 58:1045-1053

42. Wiemuth D, Gründer S: The pharmacological profile of brain liver intestine $\mathrm{Na}+$ channel: inhibition by diarylamidines and activation by fenamates. Mol Pharmacol 2011, 80:911-919.

43. Filshie BK, Flower NE: Junctional structures in hydra. J Cell Sci 1977, 23:151-172.

44. Teka IA, Kazibwe AJ, El-Sabbagh N, Al-Salabi MI, Ward CP, Eze AA, Munday JC, Maser $P$, Matovu E, Barrett MP, de Koning HP: The diamidine diminazene aceturate is a substrate for the high-affinity pentamidine transporter: implications for the development of high resistance levels in trypanosomes. Mol Pharmacol 2011, 80:110-116.

45. Ward CP, Wong PE, Burchmore RJ, de Koning HP, Barrett MP: Trypanocidal furamidine analogues: influence of pyridine nitrogens on trypanocidal activity, transport kinetics, and resistance patterns. Antimicrob Agents Chemother 2011, 55:2352-2361.

46. Benos DJ, Reyes J, Shoemaker DG: Amiloride fluxes across erythrocyte membranes. Biochim Biophys Acta 1983, 734:99-104.

47. Steinmetz PR, Kraus JE, Larroux C, Hammel JU, Amon-Hassenzahl A, Houliston E, Worheide G, Nickel M, Degnan BM, Technau U: Independent evolution of striated muscles in cnidarians and bilaterians. Nature 2012, 487:231-234.

48. Martinez DE, Iniguez AR, Percell KM, Willner JB, Signorovitch J, Campbell RD: Phylogeny and biogeography of Hydra (Cnidaria: Hydridae) using mitochondrial and nuclear DNA sequences. Mol Phylogenet Evol 2010, 57:403-410

49. Benos DJ, Prusch RD: Osmoregulation in fresh-water Hydra. Comp Biochem Physiol A 1972, 43A:165-171. 
50. Darriba D, Taboada GL, Doallo R, Posada D: ProtTest 3: fast selection of best-fit models of protein evolution. Bioinformatics 2011, 27:1164-1165.

51. Guindon S, Dufayard JF, Lefort V, Anisimova M, Hordijk W, Gascuel O: New algorithms and methods to estimate maximum-likelihood phylogenies: assessing the performance of PhyML 3.0. Syst Biol 2010, 59:307-321.

52. Guder C, Pinho S, Nacak TG, Schmidt HA, Hobmayer B, Niehrs C, Holstein TW: An ancient Wnt-Dickkopf antagonism in Hydra. Development 2006, 133:901-911.

53. Madeja M, Musshoff U, Speckmann EJ: Improvement and testing of a concentration-clamp system for oocytes of Xenopus laevis. J Neurosci Methods 1995, 63:211-213.

doi:10.1186/s12915-014-0084-2

Cite this article as: Assmann et al: The comprehensive analysis of DEG/ENaC subunits in Hydra reveals a large variety of peptide-gated channels, potentially involved in neuromuscular transmission. BMC Biology 2014 12:84

\section{Submit your next manuscript to BioMed Central and take full advantage of:}

- Convenient online submission

- Thorough peer review

- No space constraints or color figure charges

- Immediate publication on acceptance

- Inclusion in PubMed, CAS, Scopus and Google Scholar

- Research which is freely available for redistribution 